\title{
A MODIFICATION OF THE USUAL METHOD OF NEEDLING THE LENS CAPSULE AFTER CATARACT EXTRACTION*
}

\author{
BY
}

\author{
BASIL LANG
}

LONDON

After the lens has been removed, and the eye is quiet, it is desirable to make a hole in the lens capsule. If no hole be made, in years to come-it may be five to ten years-the visual acuity will begin to be reduced. At a date so distant from the extraction, the capsule will have lost its elasticity and the operation will be difficult since the incision will not gape and any flap cut will fall back into its original place and continue to occlude the pupillary area. Therefore, the operation of needling should be performed even if the pupil be occupied by thin translucent capsule through which the patient can see quite well.

The operation in such a case will not, in all probability, improve the patient's acuity of vision to any considerable extent, and it is performed, not for its immediate, but for its remote beneficial effect.

In days gone by surgeons often remained content with a result of $6 / 18$ or even less, quoting that they had known eyes, on which a perfectly successful cataract operation had been performed, lost simply because the surgeon was not content to let well alone. That eyes were, and are, occasionally lost is unfortunately only too true. The reason for this loss is two-fold. The first, sepsis. The second, glaucoma, due to the angle becoming blocked either by vitreous or exudate from a chronic cyclitis or both.

The sepsis is not the result, in the majority of cases, of faulty sterilizing of the needle. It results from the operation track becoming infected with organisms from the conjunctival sac, and it is probable that it can only occur if the needle be made to enter the anterior chamber by passing it directly through the cornea. If the organisms be very virulent, they may attack the cornea itself at the point of entry and cause an infected ulcer, which rapidly spreads along the track. But it is probable that suppuration usually follows the growth of less virulent bacteria on traces of lens substance that may escape from the anterior chamber through the incision. It is for this reason that, in the days before the condition of the conjunctival sac was bacteriologically investigated, "curette evacuation" caused such anxiety to the surgeon.

This source of accident is eliminated if the needle be passed subconjunctivally into the anterior chamber. The bacteria which

*A communication read before the Section of Ophthalmology, Royal Society of Medicine, February 8, 1924. 
can thrive and multiply in the damaged avascular cornea, live for a negligible length of time in the vascular subconjunctival tissue and the track is so tortuous that bacterial spread into the globe is impossible.

As a further means of making assurance doubly sure, the surface of the conjunctiva may be painted at the point of entry of the needle with a 1 per cent. solution of silver nitrate.

So much for sepsis.

Whenever vitreous gets into the anterior chamber there is a liability to glaucoma. This is the reason why dislocation of the lens, either accidental or intentional, occasionally ends so disastrously in glaucoma. It is for this reason that I cannot bring myself to advise any patient to have his lens removed in its capsule either by pressure or suction, since in either case the vitreous is left unsupported.

If the needle be passed far back towards the centre of the globe in an attempt to break some tough strand of capsule, the vitreous may be broken up and some come forwards into the aqueous.

If at the same time the anterior chamber be lost as the result of the blade of the needle being too broad the vitreous itself may come forwards into the anterior chamber.

If much energy be expended and the capsule be divided by a process of pulling on it with the side of the blade or the shaft of the needle rather than by cutting it with the edge, considerable inflammatory change may occur in the ciliary body and a chronic cyclitis be started. The aqueous containing the inflammatory exudate throws further difficulties on filtration and a glaucoma is liable to result.

It is to avoid these complications that I have evolved the following modification :

First I acquire a needle of suitable proportions. The blade of the needle, on being passed through the cornea, makes an incision. If the needle be passed further into the globe, the shaft comes to lie between the lips of the incision. If the shaft be the correct size, it just fills up the hole and prevents the aqueous escaping. For this reason, if the diameter of the shaft be considered as "1," the width of the blade should be " $1 \frac{1}{2}$." It is only with the greatest difficulty that a needle can be purchased of this form. The blade is usually 2 or $2 \frac{1}{2}$ times the diameter of the shaft (see table).

$\begin{array}{lllc} & \text { Shaft. } & \text { Blade. } & \text { Blade-shaft. } \\ \text { 1. } & 0.4 \mathrm{~mm} . & 0.75 \mathrm{~mm} . & 1.9 \\ 2 . & 0.5 \mathrm{~mm} . & 0.8 \mathrm{~mm} . & 1.6 \\ 3 . & 0.3 \mathrm{~mm} . & 0.7 \mathrm{~mm} . & 2.3\end{array}$

Measurements made by mounting the needles on the mechanical stage of a microscope fitted with a vernier. The three needles were taken at random from a box of needles as supplied by the makers. 
Instrument makers make them of these dimensions because they state there is a demand for a needle that will still make a hole in the cornea large enough for the shaft to pass through, after it has been re-sharpened and re-set once or twice.

When such a needle is used (see Fig. A), the aqueous flows through the triangular channels on each side of the shaft (shaded area) and the anterior chamber is more or less rapidly lost and the eye is jeopardized. A new needle costs six shillings and sixpence. With care it may be used a dozen times, so long as it is not blunted by being tested each time before use on the test leather. Economy, when one is dealing with the whole welfare of the eye, seems hardly justifiable.

A Saunders's shaft is conical; a Bowman's cylindrical. A

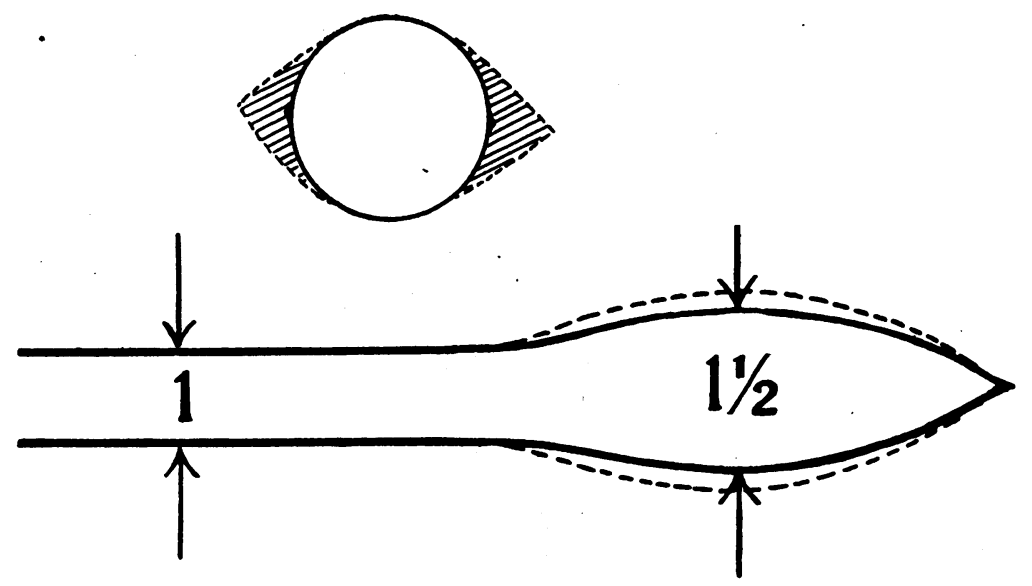

FIG. A.

Bowman's should completely fill up the hole made by the blade. For this reason I am in the habit of using a Bowman's stopped needle. I never use a Ziegler's knife. I have never convinced myself of its superiority over the needle, and it is much rarer to find one with the shaft of the correct size than it is to find a properly proportioned Bowman.

I perform the operation as soon as the eye is white, so long as there is no K.P. I have done it on the tenth day, but I think that the fourteenth is probably the earliest time at which it should be done, having regard to the fact that the wound is not solidly healed by the tenth day. The sooner the operation is done, the easier it is to do; not only does the capsule cut more easily but the incision in it gapes more readily.

I never divide the capsule if there is any quantity of unabsorbed 
lens matter. I believe that the lens matter does not absorb well in the presence of vitreous. If there is much lens matter unabsorbed at the time that the eye is white, I pass the needle into the anterior chamber in the manner about to be described and rotating the shaft through $90^{\circ}$ I move the blade "paddle fashion" in the anterior chamber stirring up any lens matter. Then I have the eye hot bathed. In the course of a few days the lens matter will have absorbed completely.

My technique is as follows :

The eye is cocainized, the speculum introduced, the room darkened and a strong light focused into the pupil.

I instruct the patient to look down and help him to do so by fixing his globe with forceps held, in the case of the right eye, in my left hand.

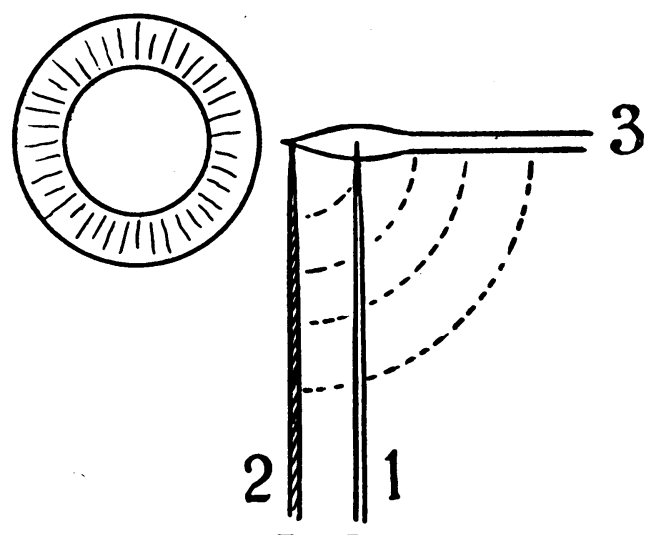

FIG. B.

My assistant touches the conjunctiva at a point some $4 \mathrm{~mm}$. from the limbus at the nine o'clock position with a small tuft of cotton wool that has been dipped into a watery 1 per cent. solution of silver nitrate.

If the needle point be passed directly into the conjunctiva at a point $4 \mathrm{~mm}$. from the limbus it is possible, and it is not very difficult, to lift up the conjunctiva on the flat of the blade and to run the needle on towards the limbus under the conjunctiva. But, as often as not, the point catches in the episcleral tissue and a certain amount of difficulty is experienced. To overcome this, I hold the needle, the point being directed towards the patient's feet, with the blade in the antero-posterior, i.e. sagittal plane (I place it with one cutting edge on the conjunctiva $4 \mathrm{~mm}$. from the limbus), the point being at the nine o'clock position (see Fig. B, position 1). I now press the edge slightly into the conjunctiva and move the needle bodily towards the limbus 
(position 2). A fold of conjunctiva is thus formed in front of the advancing blade. When the needle has reached the limbus, I twist the blade over, keeping the point stationary. The blade of the needle is now in the plane of the iris, the shaft of the needle lying in a radial direction from the centre of the cornèa (position 3). I pass the instrument through the limbus, on just in front of the iris, into the pupillary area. I have already selected a thin piece of capsule to cut. I never attempt to cut a strand. If one attempts to do so, one may pull on the ciliary body which will hurt the patient certainly after the cocain has passed off if it does not at the time, and is liable to set up a cyclitis. By raising the handle I pass the point backwards and pierce the capsule; I run the blade on just under the capsule and then by depressing the handle, I pierce the capsule a second time in the three o'clock or four o'clock position according to the position of any strands. I now twist the blade slightly and cut upwards towards the twelve o'clock position, forwards into the anterior chamber. The resulting incision is sometimes straight, sometimes curved. I observe what retraction results. This first cut rarely divides anything more than the anterior layer of the capsule. In order to divide the posterior layer it is necessary partially to withdraw the needle and to repeat the operation, but this time the handle will have to be raised quite considerably in order to pick up the posterior capsule.

In order to see what one is doing the room must be dark and the focal illumination good, else the contrast will not be good enough to enable one to see the gossamer-like posterior capsule.

If the needle be of the correct proportions, none of the aqueous will escape from the anterior chamber and the capsule may be cut three or four times if necessary.

But so long as the hole is central only a small one is necessary. It is quite possibly disadvantageous to make a large hole, for the capsule probably does good in the periphery by holding the vitreous in position.

The advantages of this modification are:

1. It is easy.

2. It avoids the possibility of sepsis.

3. It obviates the possibility of glaucoma.

4. It eliminates the necessity of stirring up the vitreous.

I do not claim that the operation is novel. Many surgeons have cut capsule by a forward movement of the blade at times.

I only wish to draw attention to the advantage of doing so and the disadvantage of not doing so, as a routine procedure. 